\title{
Tratamento cirúrgico de fratura do seio frontal utilizando enxerto de pericrânio para obliteração de ducto nasofrontal: relato de caso
}

Surgical treatment of frontal sin fracture using pericraneous graft for nasofrontal duct obliteration: clinical case Tratamiento quirúrgico de la fractura de seno frontal utilizando injerto pericráneo para la obliteración del conducto nasofrontal: reporte de caso Kaiane Tavares PONTES

Taysnara Ismaeley de ANDRADE

Francisco Rikilly de ARAÚJO

Joelma Silva de ANDRADE

Diogo Luiz Bastos BRAINER

Hospital Regional do Agreste (HRA), Residência em Cirurgia e Traumatologia Bucomaxilofacial, UPE - Universidade de Pernambuco 54.756-220 Tabatinga - Camaragibe - PE, Brasil

\section{Resumo}

Introdução: Dentre as fraturas em face destacam-se as fraturas em seio frontal, que podem resultar em dano estético. Essas fraturas podem acometer a parede anterior isoladamente ou em associação à parede posterior. A integridade do seio frontal tem importância para o paciente do ponto de vista estético e funcional. Objetivo: Este estudo tem por finalidade apresentar um caso clínico de uma vítima de acidente motociclístico, que resultou em fratura de osso frontal, associada à fratura naso-órbito-etmoidal. Relato De Caso: O paciente, do gênero masculino, 20 anos de idade, deu entrada em um Hospital de Emergência, em Pernambuco, após trauma em face. Ao exame físico observouse afundamento em região frontal, aumento da distância intercantal e desvio nasal. Ao exame tomográfico notou-se fratura naso-órbitoetmoidal associada à fratura de osso frontal, havendo deslocamento da parede anterior do seio frontal, com preservação da parede posterior. Optou-se por intervenção cirúrgica, através de acesso coronal e realizou-se a obliteração do ducto nasofrontal com enxerto livre de pericrânio e fixação da parede anterior do seio frontal; além de redução dos ossos próprios do nariz. No pós-operatório, observou-se projeção anteroposterior em região frontal adequada, melhora da distância intercantal e da arquitetura nasal. O paciente continuou sendo acompanhado durante um mês em ambulatório, evoluindo sem intercorrências. Conclusão: Pode-se concluir que o tratamento de fraturas em terços superior e médio de face constitui um desafio ao cirurgião buco-maxilo-facial, mas que um plano de tratamento adequado resulta em bons resultados para o paciente.

Descritores: Seio Frontal; Face; Fraturas Ósseas.

\section{Abstract}

Introduction: Among the fractures in the face, fractures in the frontal sinus stand out, which can result in aesthetic damage. These fractures can affect the anterior wall alone or in association with the posterior wall. The integrity of the frontal sinus is important for the patient from the aesthetic and functional point of view. OBJECTIVE: This study aims to present a clinical case of a motorcycle accident victim, which resulted in a frontal bone fracture associated with a naso-orbital-ethmoid fracture. Case Report: The male patient, 20 years old, was admitted to an Emergency Hospital, in Pernambuco, after trauma to the face. On physical examination, sagging in the frontal region, increased intercantal distance and nasal deviation were observed. CT scan revealed a naso-orbital-ethmoidal fracture associated with a fracture of the frontal bone, with displacement of the anterior wall of the frontal sinus, with preservation of the posterior wall. We opted for surgical intervention through coronal access and the nasofrontal duct was obliterated with a pericranium-free graft and fixation of the frontal sinus anterior wall; in addition to reducing the bones of the nose. In the postoperative period, anteroposterior projection was observed in an adequate frontal region, improvement of intercantal distance and nasal architecture. The patient continued to be followed up for one month on an outpatient basis, progressing uneventfully. Conclusion: It can be concluded that the treatment of fractures in the upper and middle thirds of the face is a challenge for the buccomaxillofacial surgeon, but that an adequate treatment plan results in good results for the patient.

Descriptors: Frontal Sinus; Face; Fractures, Bone.

\section{Resumen}

Introducción: Entre las fracturas en la cara, destacan las fracturas en el seno frontal, que pueden provocar daños estéticos. Estas fracturas pueden afectar la pared anterior sola o en asociación con la pared posterior. La integridad del seno frontal es importante para el paciente desde el punto de vista estético y funcional. Objetivo: Este estudio tiene como objetivo presentar un caso clínico de una víctima de accidente de motocicleta, que resultó en una fractura ósea frontal asociada con una fractura naso-orbital-etmoidea. Caso Clínico: El paciente masculino, de 20 años, ingresó en un Hospital de Urgencias, en Pernambuco, luego de un traumatismo en la cara. En el examen físico, se observó flacidez en la región frontal, aumento de la distancia intercantal y desviación nasal. El examen tomográfico reveló una fractura nasoorbital-etmoidal asociada con una fractura del hueso frontal, con desplazamiento de la pared anterior del seno frontal, con preservación de la pared posterior. La intervención quirúrgica se eligió a través del acceso coronal y el conducto nasofrontal se obliteró con un injerto libre de pericráneo y la fijación de la pared anterior del seno frontal; Además de reducir los huesos de la nariz. En el postoperatorio, se observó proyección anteroposterior en una región frontal adecuada, mejoría de la distancia intercantal y arquitectura nasal. Se siguió al paciente durante un mes de forma ambulatoria, progresando sin incidentes. Conclusión: Se puede concluir que el tratamiento de fracturas en los tercios superior y medio de la cara es un desafío para el cirujano buccomaxilofacial, pero que un plan de tratamiento adecuado resulta en buenos resultados para el paciente.

Descriptores: Seno Frontal; Cara; Fracturas Óseas.

\section{INTRODUÇÃO}

O osso frontal é composto de três camadas, sendo estas, externa ou camada cortical; a intermediária, que é vascularizada e chamada de díploe; e a interna, que é mais delgada. No osso frontal localiza-se o Seio Frontal, cavidade óssea, pneumática, com forma triangular, com a maior porção no assoalho da órbita. A fina parede posterior separa o seio das meninges e do lobo frontal do cérebro $^{1-3}$

Fraturas envolvendo o terço superior da face têm alto potencial para complicações e seu manejo ainda é controverso em certas situações ${ }^{1-3}$. A fratura do osso frontal é uma lesão rara quando comparada aos demais tipos de fraturas faciais. Ela normalmente está associada a traumas de grande impacto, como os causados por acidentes automotores ou agressões físicas. É comum a associação desta fratura àquelas do complexo naso-órbito-etmoidal ${ }^{2,4,5}$.

Quando ocorrem, as fraturas do osso frontal podem acometer anatomicamente as paredes anterior 
e/ou posterior, havendo ou não o envolvimento do ducto nasofrontal. Epidemiologicamente, observa-se que fraturas envolvendo apenas a parede anterior do seio frontal são mais comuns de acontecer ${ }^{2,4}$.

$\mathrm{O}$ tratamento das fraturas de osso frontal tem por objetivo, basicamente, a prevenção de infecções, cuidados com o conteúdo intracraniano, além da restauração da função e estética. A escolha do acesso à fratura e os materiais utilizados para fixação varia de acordo com o tipo de fratura presente, ficando a critério do cirurgião o planejamento individual para cada caso ${ }^{6}$.

Este estudo tem por finalidade apresentar um caso clínico de um paciente, vítima de acidente motociclístico, que resultou em fratura de osso frontal, a qual foi tratada cirurgicamente através da utilização de enxerto de pericrânio para obliteração do ducto nasofrontal e fixação dos fragmentos com placas, tela e parafusos de titânio, através do acesso coronal. O artigo objetiva, ainda, citar a comum associação de fraturas de osso frontal com fraturas naso-órbito-etmoidais.

\section{CASO CLÍNICO}

Paciente do sexo masculino, melanoderma, 20 anos de idade, na emergência de um Hospital de Emergência, no estado de Pernambuco, após ser vítima de acidente motociclístico. No momento do atendimento encontrava-se consciente e orientado, Escala de Coma de Glasgow 15, com pupilas isocóricas e fotorreagentes, eupneico, normocorado, verbalizando e deambulando. Ao exame físico observou-se ferimentos corto-contusos em regiões supraorbital do lado direito e nasal, edema periorbital bilateral, afundamento em região frontal, discreta epistaxe, além de aumento da distância intercantal (Figura 1). Ao exame de imagem (Tomografia Computadorizada) notou-se fratura naso-órbitoetmoidal associada à fratura de osso frontal (havendo deslocamento da parede anterior do seio frontal, com preservação da parede posterior) (Figuras 2 e 3). O paciente evoluiu com obstrução nasal e desvio de dorso nasal no pré-operatório.

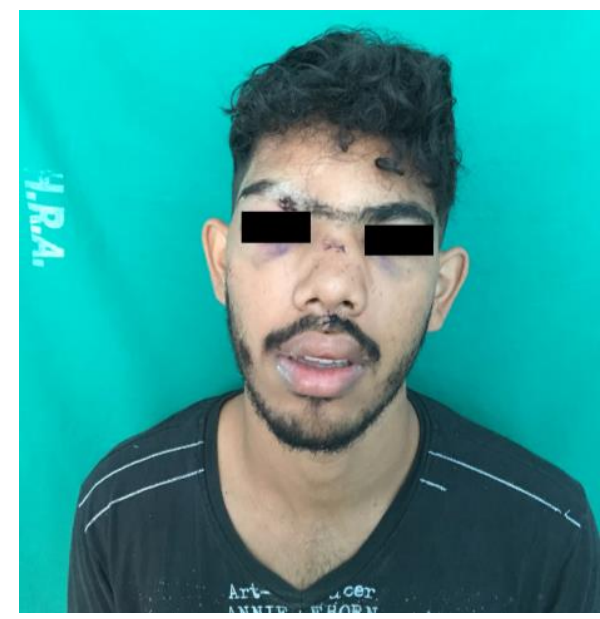

Figura 1: Paciente no pré-operatório.
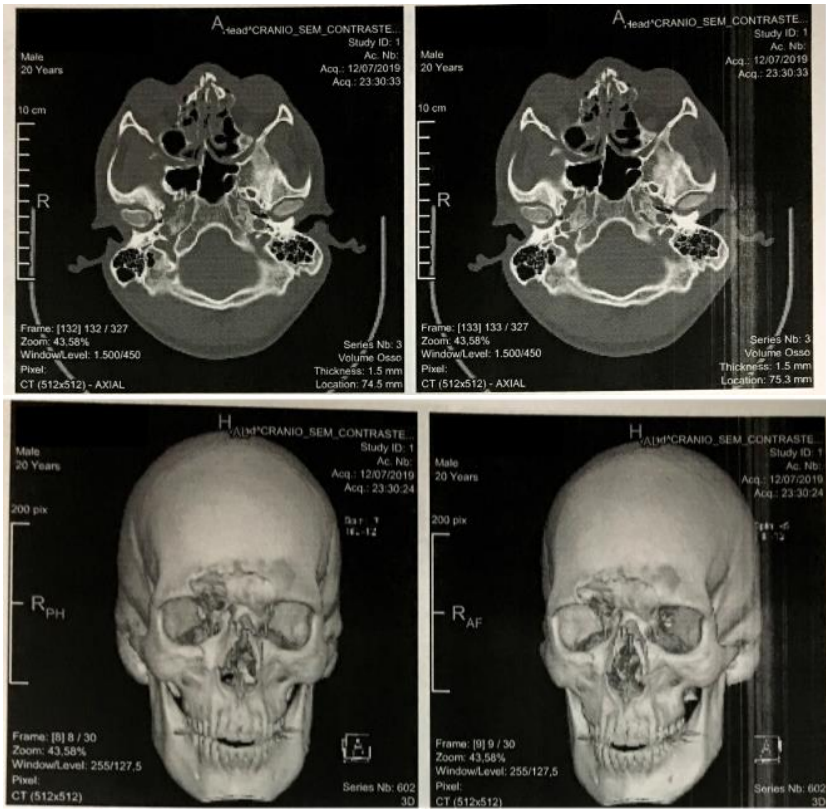

Figura 2: Tomografia computadorizada mostrando fratura naso-órbitoetmoidal.
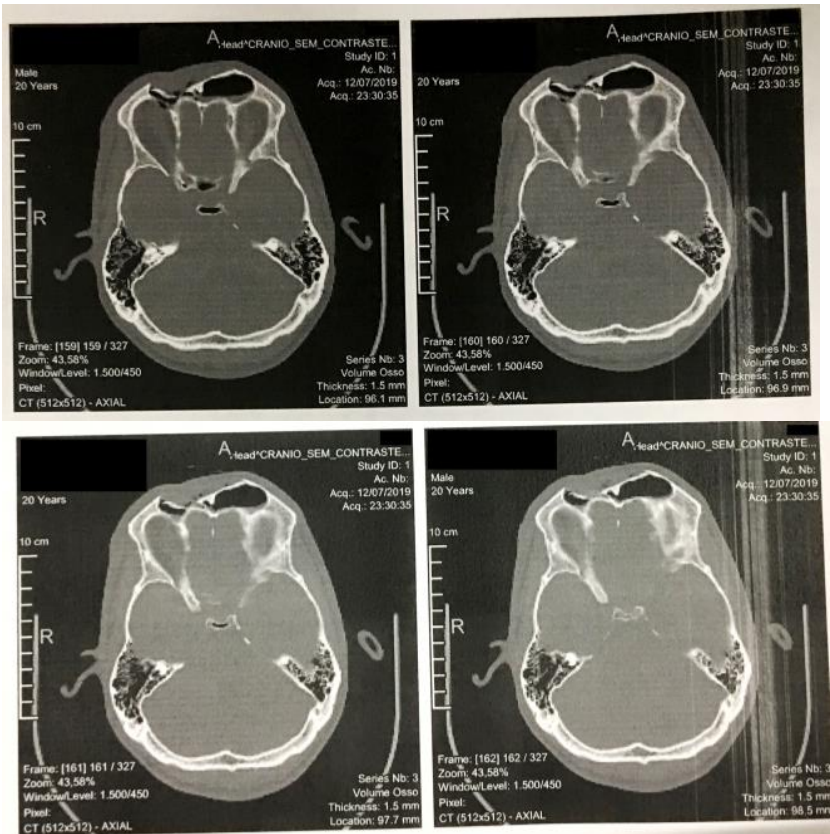

Figura 3:Tomografia computadorizada mostrando fratura de parede anterior do seio frontal.

O plano de tratamento consistiu em intervenção cirúrgica, sob anestesia geral, com intubação orotraqueal, e o acesso coronal foi realizado para visualização das fraturas do terço superior da face (Figura 4). Realizou-se, então, a curetagem do seio frontal, seguida por teste para avaliar a patente do ducto nasofrontal, durante o qual foi constatado que o mesmo se encontrava sem função. Diante disso, optou-se pela realização de obliteração do ducto nasofrontal com retalho de pericrânio (Figura 5), seguida pela fixação da parede anterior do seio frontal com placas, tela e parafusos de titânio do sistema $2.0 \mathrm{~mm}$. Além disso, realizou-se a colocação de enxerto autógeno com fragmentos da própria fratura na região fixada (Figura 6). Ainda durante a cirurgia foi feita a redução incruenta dos 
ossos próprios do nariz e instalação de tampão nasal anterior, a fim de manter a arquitetura nasal após redução. Por fim, instalou-se um dreno a vácuo na região frontal e as suturas foram realizadas.

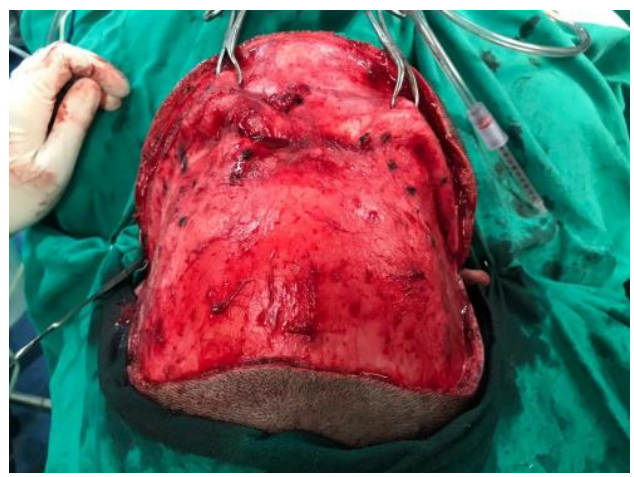

Figura 4: Acesso coronal para exposição cirúrgica.

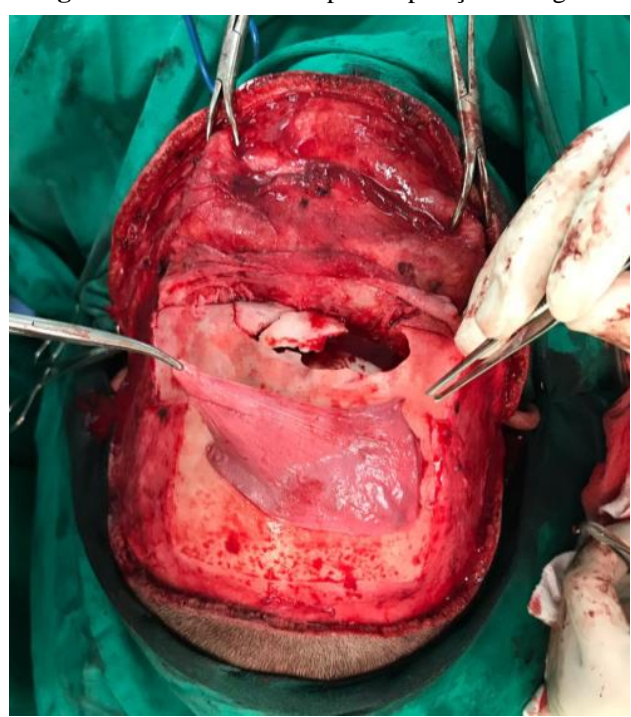

Figura 5: Retalho de pericrânio para obliteração do ducto do nasofrontal.

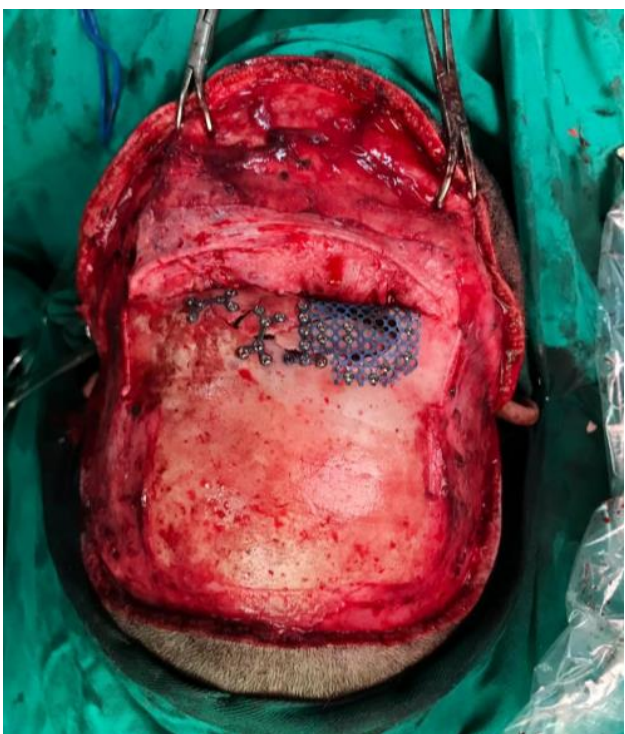

Figura 6: Fixação das fraturas com placas do sistema $2.0 \mathrm{~mm}$ e tela de titânio.

No pós-operatório, observou-se uma melhora da distância intercantal; redução da fratura dos ossos próprios do nariz mantida após remoção do tampão nasal anterior, com diminuição das queixas do paciente em relação à função; projeção frontal anteroposterior adequada, sem $\mathrm{o}$ afundamento observado previamente. $\mathrm{O}$ dreno a vácuo foi removido $24 \mathrm{~h}$ após a cirurgia.

O paciente recebeu alta $48 \mathrm{~h}$ após a cirurgia e foi orientado à realização de retornos ambulatoriais no mesmo hospital onde foi tratado. $\mathrm{O}$ mesmo apresentou boa recuperação, sem intercorrências, dentro dos padrões esperados em ternos funcionais e estéticos. Paciente seguiu de alta ambulatorial após um mês da cirurgia.

DISCUSSÃO

As fraturas de osso frontal, normalmente, são originadas através de traumas de alta energia, como acidentes com veículos automotores e agressão física, estando muitas vezes, associadas com fraturas no terço médio de face ${ }^{3,4,7,8}$ Observa-se que o sexo masculino é responsável pela maior parte dos $\operatorname{casos}^{7-10}$.

Quanto ao tratamento, Lessa $^{4}$ e Silva ${ }^{8}$ concordam que em pacientes com fratura de parede anterior, sem deslocamento ou com deslocamento e sem alterações clínicas, pode-se optar por tratamento conservador. Enquanto que nos casos em que há necessidade de intervenção cirúrgica, os autores concordam que três estruturas devem ser levadas em consideração: parede anterior, parede posterior e ducto nasofrontal. Essas estruturas irão influenciar na escolha do acesso cirúrgico, necessidade de fixação, seleção de material, indicações para cateterização do ducto nasofrontal, além de tecido para obliteração do seio e do ducto.

A cirurgia para fraturas de seio frontal visa restabelecimento da estética e função. $\mathrm{O}$ tratamento incorreto pode gerar complicações como mucocele, meningite, abscessos cerebrais, encefalite, piocele e sinusite crônica, como visto na literatura ${ }^{10,11}$. Em alguns casos é necessário realizar o preenchimento do seio, juntamente com obliteração do ducto nasofrontal. $\mathrm{O}$ acesso coronal corresponde à indicação mais adequada para visualização de fraturas do terço superior da face, tendo sido o mais utilizado na maioria dos estudos $9,10,12$.

Em seu estudo, Fernandes ${ }^{10}$ optou por realização de craniotomia, obliteração do ducto nasofrontal com "chips ósseos" e retalho de pericrânio, seguido por redução e fixação das fraturas. Enquanto Santos ${ }^{11}$ descreve em seu caso clínico, a obliteração do seio de ducto nasofrontal com retalho da fáscia do músculo temporal e Gelfoam (gelatina absorvível), seguida por fixação das fraturas associadas. Em nosso estudo, foi utilizado enxerto de pericrânio com a finalidade de obliterar o ducto nasofrontal, com posterior redução e fixação das fraturas.

\section{CONCLUSÃO}

Pode-se concluir que a escolha da técnica cirúrgica a ser empregada dependerá da gravidade e 
extensão da fratura e das repercussões clínicas apresentadas pelo paciente. Idealmente, a cirurgia deve basear-se na exploração e reparo das lesões, de forma que o paciente tenha sua estética restituída, quando for o caso, e evolua sem complicações do ponto de vista funcional no pós-operatório.

\section{REFERÊNCIAS}

1. Silva JJ, Neto AR, Pereira AM, Correia V, Lira AA. Fratura tardia de seio frontal: relato de caso. Rev Cir Traumatol buco-maxilo-fac. 2005; 5(3):51-6.

2. Montovani JC, Nogueira EA, Ferreira FD, Lima Neto AC, Nakajima V. Cirurgia das fraturas do seio frontal: estudo epidemiológico e análise de técnicas. Rev Bras Otorrinolaringol. 2006; 72(2):204-9.

3. Conci RA, Martins JRP, Tomazi FH, Sbardelotto BM, Sirena Neto L, Oliveira GR. Tratamento Cirúrgico de fratura de seio frontal. Surgical Treatment of Frontal Sinus Fracture. Rev Cir Traumatol Buco-Maxilo-Fac. 2012;(12):31-36.

4. Lessa ES, Cruz RL, Costa MJM, Magalhaes GE, Braune AS. Fraturas do seio frontal: conduta em relação ao ducto nasofrontal. Rev Bras Cir Plást. 2010;25(supl):1-102.

5. Melo, RB, Hage CA, Carneiro NCM, Xavier TB, Fonseca WLM, Ferreira DP. Tratamento cirúrgico de fratura de parede anterior de seio frontal decorrente de acidente desportivo: relato de caso clínico. Rev Odontol Bras Central 2016;25(72).

6. Doonquah L, Brown P, Mullings W. Management of frontal sinus fractures. Oral Maxillofac Surg Clin North Am. 2012;24(2):265-74.

7. Melo MFS, Zanettini LMS, Lukschal LF, Silveira RL, Amaral MBF. Correção de fratura frontonaso-órbito-etmoidal: passos cirúrgicos para resultado estético. Cir Traumatol Buco-MaxiloFac. 2015;15(1):33-40.

8. Silva JR, Mourão CFAB, Rocha Júnior HV, Magacho LF, Moraes GFD, Homsi N. Inversão do segmento fraturado para tratamento das sequelas de fratura do seio frontal. Rev Col Bras Cir 2016; 43(6):472-75.

9. Macedo TFO, Lima RFF, Toledo IC, Cavalcante WC, Santos JN. Tratamento de fratura da parede anterior do seio frontal com técnica minimamente invasiva: relato de caso clínico. Rev Bras Cir Cabeça Pescoço. 2017;46(3):105-8.

10. Fernandes BDR, Mandarino S, Gomes-Ferreira PHS, Palin LP, Zorzi Coléte J, Ribeiro J, et al. Tratamento de fratura do seio frontal, por meio de cranialização, obliteração, redução e fixxação das fraturas: relato de caso clínico. Arch Health Invest. 2018;7 (Spec Iss 3):451.

11. Santos MBP, Cavalieri I, Araújo MM, Vale DS, Breda Júnior, MA. Inversão do segmento fraturado para tratamento das sequelas de fratura do seio frontal. Rev Col Bras Cir. 2016; 43(6):472-75.

12. Jardim EC, Santiago Júnior JF, Guastaldi FPS, Magro Filho O, Jardim Júnior, EG. Fratura do seio frontal: relato de caso. Rev Odontol Araçatuba 2010;31(2):35-39.

\section{CONFLITO DE INTERESSES}

Os autores declaram não haver conflitos de interesse.

\section{AUTOR PARA CORRESPONDÊNCIA}

\section{Kaiane Tavares Pontes}

Av. General Newton Cavalcanti, 1650 - Tabatinga

54.756-220 Camaragibe - PE, Brasil

email: kaianetavares@hotmail.com 